\title{
Real-Time Monitoring of Neural Differentiation of Human Mesenchymal Stem Cells by Electric Cell-Substrate Impedance Sensing
}

\author{
Hyo Eun Park, ${ }^{1}$ Donghee Kim, ${ }^{1}$ Hyun Sook Koh, ${ }^{1}$ Sungbo Cho, ${ }^{2}$ Jung-Suk Sung, ${ }^{3}$ \\ and Jae Young Kim ${ }^{1}$ \\ ${ }^{1}$ Department of Biological Science, Gachon University of Medicine and Science, Incheon 406-799, Republic of Korea \\ ${ }^{2}$ Department of Biomedical Engineering, Gachon University of Medicine and Science, Incheon 406-799, Republic of Korea \\ ${ }^{3}$ Department of Life Science, Dongguk University-Seoul, Seoul 100-715, Republic of Korea
}

Correspondence should be addressed to Jae Young Kim, jykim85@gachon.ac.kr

Received 21 December 2010; Revised 23 February 2011; Accepted 2 April 2011

Academic Editor: Andre Van Wijnen

Copyright ( $) 2011$ Hyo Eun Park et al. This is an open access article distributed under the Creative Commons Attribution License, which permits unrestricted use, distribution, and reproduction in any medium, provided the original work is properly cited.

\begin{abstract}
Stem cells are useful for cell replacement therapy. Stem cell differentiation must be monitored thoroughly and precisely prior to transplantation. In this study we evaluated the usefulness of electric cell-substrate impedance sensing (ECIS) for in vitro real-time monitoring of neural differentiation of human mesenchymal stem cells (hMSCs). We cultured hMSCs in neural differentiation media (NDM) for 6 days and examined the time-course of impedance changes with an ECIS array. We also monitored the expression of markers for neural differentiation, total cell count, and cell cycle profiles. Cellular expression of neuron and oligodendrocyte markers increased. The resistance value of cells cultured in NDM was automatically measured in real-time and found to increase much more slowly over time compared to cells cultured in non-differentiation media. The relatively slow resistance changes observed in differentiating MSCs were determined to be due to their lower growth capacity achieved by induction of cell cycle arrest in G0/G1. Overall results suggest that the relatively slow change in resistance values measured by ECIS method can be used as a parameter for slowly growing neural-differentiating cells. However, to enhance the competence of ECIS for in vitro real-time monitoring of neural differentiation of MSCs, more elaborate studies are needed.
\end{abstract}

\section{Introduction}

Due to their long-term self-renewal capacity and multilineage differentiation potential, stem cells have been considered as useful replacement material to heal cellular injuries caused by trauma, infection, and genetic diseases. However, stem cells must be differentiated into the appropriate cell types prior to transplantation for cell replacement therapy; otherwise, the risk of tumor formation cannot be ruled out [1]. In addition, the purity and yield of differentiated cells are critical for successful stem cell therapy [1]. For these reasons, monitoring the process of in vitro stem cell differentiation is important.

In general, reverse transcription polymerase chain reaction (RT-PCR), Northern and Western blotting, immunofluorescence assays, and flow cytometric analysis for particular markers have been applied to detect in vitro stem cell differentiation. In addition, genomic and proteomic analysis are also sometimes used. However, all the methods mentioned are labor-intensive multistep processes, which are end-point assays that offer only a snapshot of what is occurring. These techniques usually involve labeling with nucleic acids or antibodies and destruction of the cells. To successfully examine dynamic cellular processes in live cells, nondestructive real-time monitoring methods are needed.

Electric cell-substrate impedance sensing (ECIS) is a noninvasive approach which has been used to analyze the morphological and electrophysiological characteristic of living cells [2, 3], cell growth $[4,5]$, cell death [6], cytotoxicity [7], cytopathy [8], and cell migration [9]. Furthermore, Cho et al. [10] and Hildebrandt et al. [11] reported the possibility of impedance measurement for 
the label-free characterization of adipogenic or osteogenic differentiation of bone marrow-derived stem cells which could then be correlated with morphological or physiological changes caused by differentiation.

In the present study, we performed neural differentiation of human umbilical cord-derived mesenchymal stem cells (hMSCs) according to a previously established differentiation protocol [12] and attempted to evaluate the usefulness of ECIS for real-time monitoring of differentiation by measuring the resistance change of the cell layer.

\section{Materials and Methods}

2.1. Cell Culture Conditions. Human mesenchymal stem cells obtained from umbilical cord matrix (hMSC-UC) (PromoCell GmbH, Heidelberg, Germany) were cultured in MSC growth medium (PromoCell) supplemented with $0.5 \mathrm{x}$ antibiotic-antimycotic (Gibco, MD, USA) in $100 \mathrm{~mm}$-cell culture dishes. Cells were incubated at $37^{\circ} \mathrm{C}$ in a humidified atmosphere containing $5 \% \mathrm{CO}_{2}$ for 7 days, and the culture medium was changed once every 2-3 days. When $80-90 \%$ of adherent cells were confluent, they were harvested with $0.05 \%$ trypsin-EDTA solution (Gibco). To determine the usefulness of ECIS for real-time monitoring of neural differentiation of hMSCs, the cells were divided into 3 groups as follows: normal growth medium (NGM), neural induction medium (NIM), and neural differentiation medium (NDM). Cells in the NGM group were maintained in NGM for $139 \mathrm{hrs}$. Cells in the NIM group were incubated in NGM for $16 \mathrm{hrs}$, then transferred to NIM consisting of NGM supplemented with $10 \mathrm{ng} / \mathrm{mL}$ basic fibroblast growth factor (b-FGF) (Invitrogen, Carlsbad, CA, USA), and then cultured for $123 \mathrm{hrs}$. Cells in the NDM group were cultured in NGM for $16 \mathrm{hrs}$, transferred to NIM and cultured for $24 \mathrm{hrs}$ and finally transferred to and maintained in NDM consisting of NGM supplemented with 1\% dimethylsulfoxide (DMSO, Sigma Aldrich Corp., St. Louis, MO, USA) and $200 \mu \mathrm{M}$ butylated hydroxyanisole (BHA, Sigma) for $99 \mathrm{hrs}$.

2.2. ECIS Assay. For the impedance characterization of hMSC behavior during neural differentiation, singleelectrode arrays with a gold layer were prepared $(8 \mathrm{~W} 10 \mathrm{E}$ +, Applied BioPhysics, Troy, NY, USA). After placing $400 \mu \mathrm{L}$ of media in the wells, the electrode arrays were allowed to equilibrate in an incubator for $30 \mathrm{~min}$. For the inoculation of cells, $400 \mu \mathrm{L}$ of cell suspension $\left(3.725 \times 10^{4}\right.$ cells per $\mathrm{mL}$ ) was transferred to each well, resulting in a final surface concentration of $1.875 \times 10^{4}$ cells per $\mathrm{cm}^{2}$. After the electrode arrays were located in the well station to interface each electrode with the measurement system (ECIS Z, Applied BioPhysics), the resistance of cells was monitored during cultivation with or without the differentiation factor. The resistance of cells was recorded every $5 \mathrm{~min}$ by applying the input current of $1 \mu \mathrm{A}$ at $16 \mathrm{kHz}$ between a small sensing electrode $(250-\mu \mathrm{m}$ diameter) and a relatively large counter electrode.

2.3. Immunocytochemistry. To evaluate the immune phenotype, hMSCs were cultured with or without NDM in 8-well plates (Lab-Tek, Nalge Nunc International, Naperville, IL, USA) and tested with an intracellular immunofluorescent staining flow analysis kit (Imgenex, San Diego, CA, USA) according to the manufacturer's protocol. For this purpose, cells were incubated with specific antibodies directed against different phenotypic markers, including: the neural precursor marker, nestin $(1: 100$, Chemicon, Temecula, CA, USA), the class III $\beta$ tubulin ( $1: 100$, Tuj-1, Santa Cruz Biotechnology, Santa Cruz, CA, USA), the astrocyte marker, GFAP ( $1: 50$, Zymed Laboratories; San Francisco, CA, USA), the oligodendrocyte marker, O4 (1:50, Chemicon), mouse IgG ( $1: 100$, Cedarlane, Hornby, Ontario, Canada), rat IgG ( $1: 100$, Jackson ImmunoResearch, West Grove, PA, USA), and mouse IgM (1:100, Chemicon). Cells present in 10 optical fields (300x) were examined under a Nikon Eclipse TE2000U microscope and analyzed with Nikon NIS Elements Basic Research software. The percentages of positive cells for each antigen were obtained and compared to the total number of cells labeled with Hoechst stains.

2.4. FACS Analysis. Human MSC-UCs were recognized by immunophenotype using monoclonal antibodies (mAbs) specific for PE-CD13, FITC-CD44, PE-CD14, FITC-CD34, PE-CD90, PE-CD73 (BD Pharmingen, San Diego, CA, US), RPE-CD105, and RPE-CD45 (AbD Serotec, Oxford, UK). For immunophenotypic analysis, hMSC-UCs were detached using trypsin/EDTA for $5 \mathrm{~min}$, then immediately washed with phosphate-buffered saline (PBS) to remove trypsin, and resuspended at $10^{6}$ cells $/ \mathrm{mL}$. Cells were stained with specific $\mathrm{mAbs}$ at $4^{\circ} \mathrm{C}$ for $30 \mathrm{~min}$ in PBS then washed and resuspended in PBS. At least, 10,000 events were analyzed using a Beckman Coulter FC500 flow cytometer (Beckman Coulter, Fullerton, CA, USA) and Beckman Coulter Cytomics CXP software (Applied Cytometry Systems, USA).

2.5. Cell Count and Cell Cycle Analysis. Cells were plated on 12-well culture plates at a density of $1.875 \times 10^{4}$ cells $/ \mathrm{cm}^{2}$. After $36 \mathrm{hrs}$ of neural differentiation, the cultures were trypsinized and the total cells were counted on a hemocytometer. Cell cycle analysis of hMSCs was performed after $24 \mathrm{hrs}$ of neural differentiation. hMSCs $\left(10^{6}\right)$ were washed with PBS 3 times, fixed with ice cold $70 \%$ ethanol, and stored at $4^{\circ} \mathrm{C}$ for a minimum of $1 \mathrm{hr}$. After 3 more washing steps with PBS, the DNA of the hMSCs was stained with propidium iodide/RNase staining buffer (BD Biosciences, Heidelberg, Germany). The samples were then incubated at room temperature for $15 \mathrm{~min}$ and analyzed by flow cytometry.

2.6. RNA Extraction and RT-PCR. Total RNA was isolated using QIAamp RNA Blood Mini Kits (Qiagen, Hilden, Germany) according to the manufacturer's protocol. The concentration and purity of the RNA were determined by reading at OD260/280. The cDNA was synthesized from $2 \mu \mathrm{g}$ of total RNA with a MMLVRT (Beams Biotech, Seongnam, Korea) using an Oligo (dT) primer (Invitrogen) at $37^{\circ} \mathrm{C}$ for $1 \mathrm{hr}$. PCR amplification was performed using the primer sets represented in Table 1. Thermocycling conditions were 


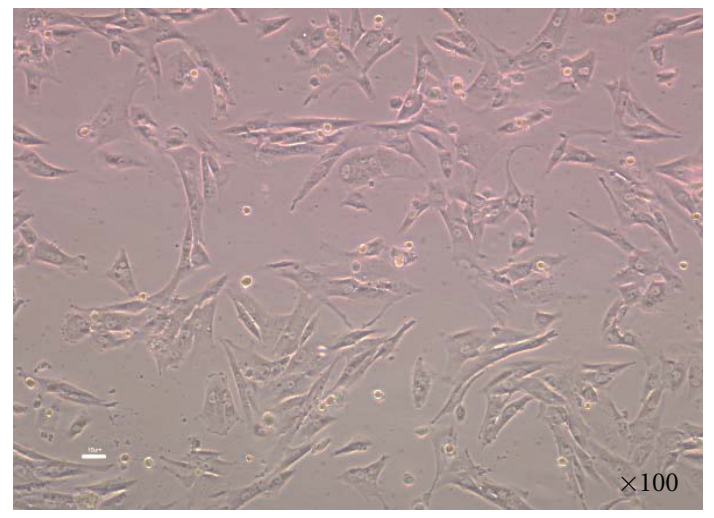

(a)
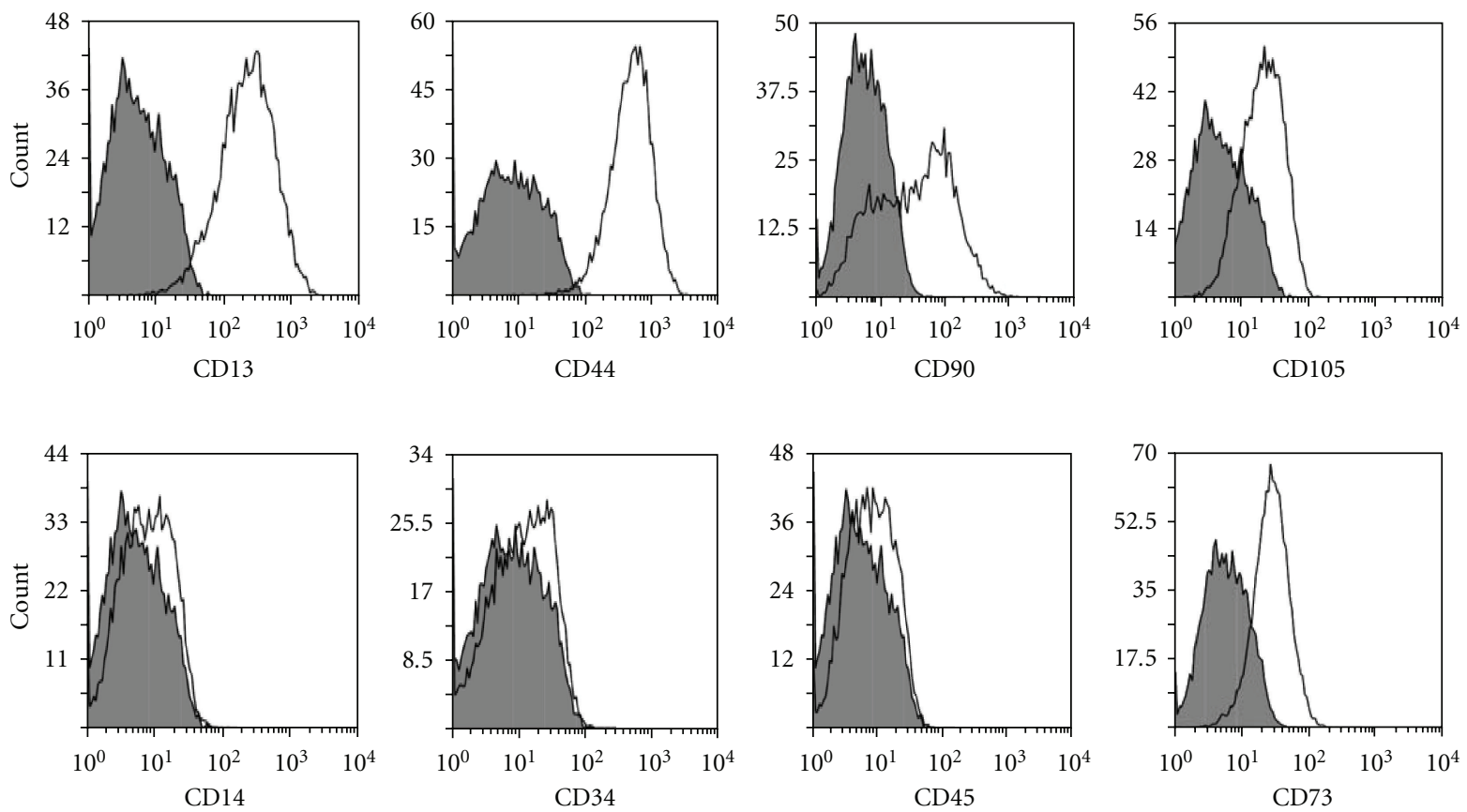

(b)

FIGURE 1: Morphological and flow cytometric characterization of primary human mesenchymal stem cells (hMSCs). (a) Morphology of primary human umbilical cord blood-derived MSC cultured in normal growth medium. hMSCs at passages 8-9 have a fibroblast-like morphology. $\times 100$. (b) Immunophenotyping results of human umbilical cord blood-derived MSCs. Cells at passages 8-9 were stained with antibodies listed in Section 2 and assayed by flow cytometry. The cells were strongly positive for MSC-specific markers, such as CD13, CD44, CD73, CD90, and CD105, while negative for the hematopoietic stem cell markers CD14, CD34, and CD45. Staining for nonspecific immunoglobulin $\mathrm{G}(\mathrm{IgG})$ isotype fluorescence used as a control is represented by gray fill.

$30 \mathrm{sec}$ at $95^{\circ} \mathrm{C}, 30 \mathrm{sec}$ at $54-65^{\circ} \mathrm{C}$, and $30 \mathrm{sec}$ at $72^{\circ} \mathrm{C}$ for $26-$ 40 cycles preceded by $10 \mathrm{~min}$ at $95^{\circ} \mathrm{C}$. PCR products were fractionated by $2 \%$ agarose gel electrophoresis, stained with LoadingSTAR (DyneBio, Seongnam, Korea), and visualized with ultraviolet light. The bands were quantitated by densitometric analysis using KODAK MI software (Rochester, NY, USA).

2.7. Statistical Analysis. Data were evaluated for statistical significance using SPSS 12.0 for Windows. Values are expressed as the mean \pm standard deviation (SD). The mean values were compared using a one-way analysis of variance
(ANOVA) followed by Scheffe's multiple comparison test to determine differences between the means.

\section{Results}

3.1. Characterization of Primary hMSCs. To determine whether umbilical cord-derived hMSCs purchased from Promocell had MSC characteristics, we examined the cells for morphologic characteristics and expression of MSC-specific markers. Figure 1(a) shows that primary MSCs display a typical fibroblast-like morphology. These cells expressed MSC specific markers such as CD13, CD44, CD105, CD90, 

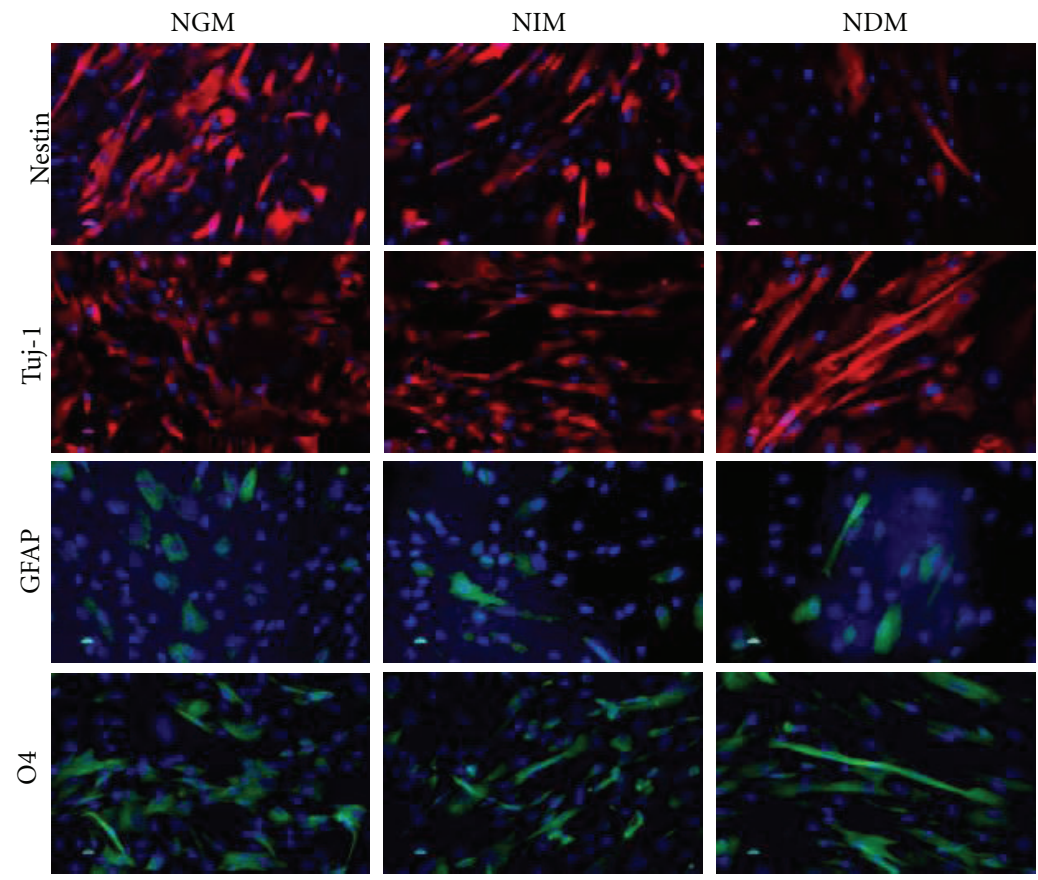

(a)
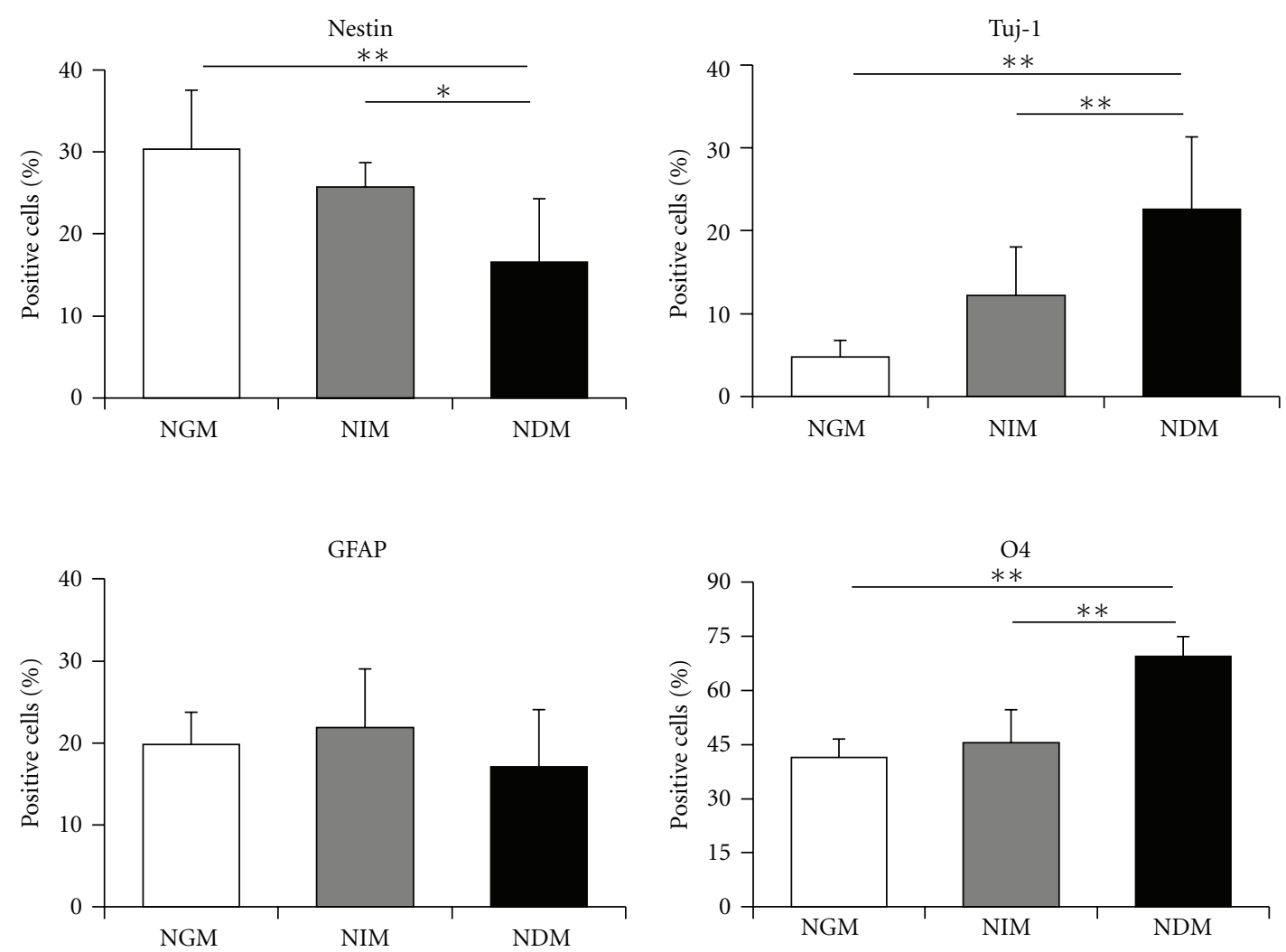

(b)

FIGURE 2: Neural marker expression of hMSCs during neural differentiation. (a) Immunocytochemical analysis of differentiated hMSC with neuronal lineage-specific antibodies ( $\times 300$ objective). Cells that had been harvested $72 \mathrm{hr}$ after changing to a NDM were stained with the primary antibodies listed in Materials and methods. Hoechst 33328 was used for nuclear counterstaining. (b) Quantification of the percentage of stained cells from two different slides (10 fields of view, except in the case of O4 with 5 fields and Tuj-1 with 8 fields). Data represent mean $\pm \mathrm{SD}$. ${ }^{*} P<.05^{* *} P<.01$. NGM: normal growth media; NIM: neural induction media; NDM: neural differentiation media. 


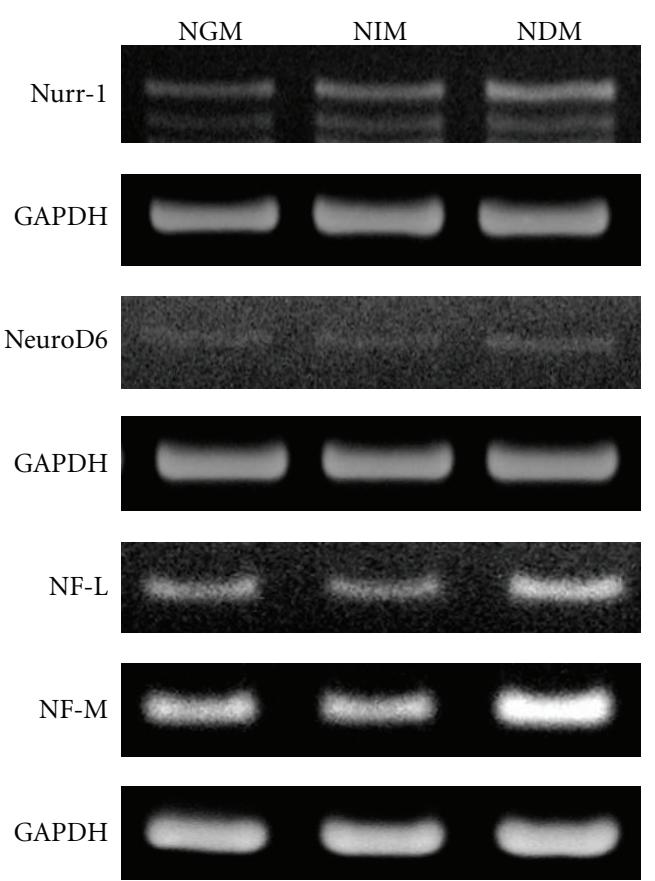

(a)
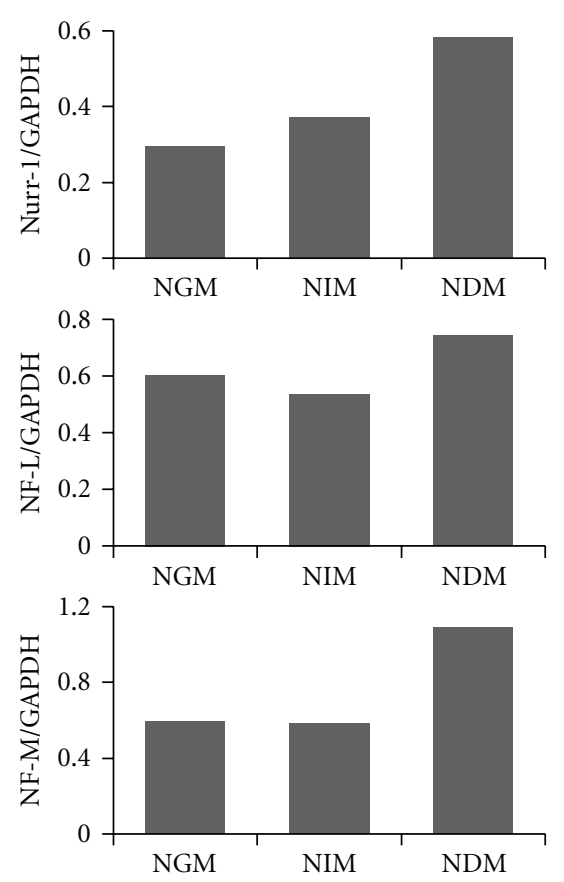

(b)

Figure 3: Neuron-specific gene expression of hMSCs during neural differentiation. (a) Cells that had been harvested 6 hr for Nurr-1, 12 hr for NeuroD6, and $48 \mathrm{hr}$ for NF-L and NF-M were used in RT-PCR assay. NGM: normal growth media; NIM: neural induction media; NDM: neural differentiation media. (b) The relative expression of Nurr-1, NF-L, and NF-M was normalized by GAPDH using densitometric analysis. Data for NeuroD6 were omitted because bands corresponding to NeuroD6 were too faint to be measured by densitometry.

TABLE 1: Sequences of the RT-PCR primers.

\begin{tabular}{ll}
\hline & Sequence $\left(5^{\prime}\right.$ to $\left.3^{\prime}\right)$ \\
\hline \multirow{2}{*}{ Nurr-1 } & AAA AGG CCG GAG AGG TCG TTT GCC \\
& TGG GTT CCT TGA GCC CGT GTC TC \\
NeuroD6 & CTG AGA ATC GGC AAG AGA CC \\
& CTG CAC AGT AAT GCA TGC CG \\
NF-L & AGA CCC GAC TCA GTT TCA C \\
& ACC TTC ACC TCC TTC TTC TT \\
NF-M & AAG CCA ATC AGA CCA GAA TA \\
& GCA GCG ATT TCT ATA TCC AG \\
GAPDH & ACA GCC TCA AGA TCA TCA GCA AT \\
& AGG AAA TGA GCT TGA CAA AGT GG \\
\hline
\end{tabular}

and CD73 but not hematopoietic stem cell markers such as CD14, CD34, and CD45 (Figure 1(b)).

3.2. Neural Marker Expression of hMSC during Neural Differentiation. We examined the in vitro neural differentiation of hMSCs cultured under 3 different conditions slightly modified from a previous protocol [12] as described in Materials and Methods. The expression of neural specific markers by cells harvested from the 3 different groups was examined by immunofluorescence assay (Figure 2). Nestin, known as a neural stem cell marker, was expressed in hMSCs cultured in NGM indicating that hMSCs are ready to respond to exogenous signals stimulating neural differentiation. Nestin expression was significantly reduced in cells cultured in NDM but not in those in NIM. The reduction of nestin expression in the cells cultured in NDM reflects their differentiation. The neuron-specific marker, Tuj-1, was expressed in cells cultured in all 3 conditions. However, the proportion of Tuj-1 expressing cells with neuron-like morphology was significantly higher in NDM than in the other culture media (Figure 2(a)). The expression level of the astrocyte marker GFAP in cells cultured in NDM was not significantly different from those in the other media. Although the oligodendrocyte marker $\mathrm{O} 4$ was expressed in the cells cultured in all 3 different media, O4-positive cells were determined to be present more in NDM than in the other culture media. Similar to microscopic findings, Tuj1-positive cell counts were higher in NDM, followed by NIM and NGM (Figure 2(b)). O4-positive cell counts were also higher in NDM than in the other media $(P<.01)$. In contrast, GFAP-positive cell counts were not different between the 3 media, and nestin-positive cell counts in NDM were twice as low as in the other groups $(P<.01)$.

Since the numbers of cells expressing the neuron-specific protein marker Tuj-1 in NDM were found to be twice as high as those in the other media, cellular mRNA expressions of several neuron-specific genes were examined by RT-PCR. As shown in Figure 3, the expression levels of neuronal markers such as Nurr-1, NF-L, and NF-M in the NDM group were relatively higher than in the other groups. Overall results indicate that hMSCs cultured in NDM were effectively induced to differentiate into neuronal cells. 


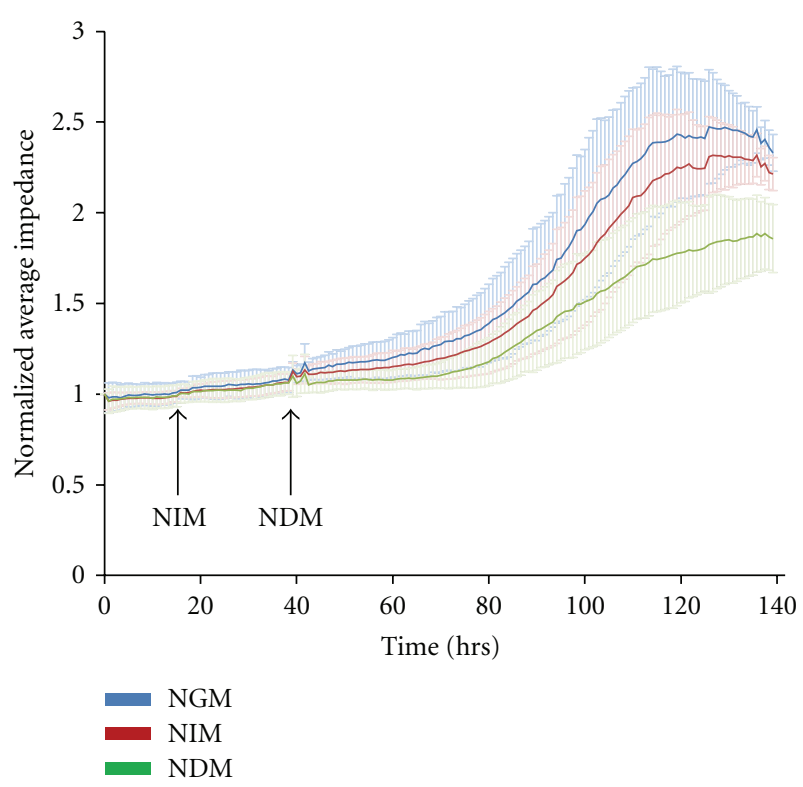

FIgURE 4: Resistance monitoring of hMSC layers during the cultivation with or without the neural differentiation factor. The resistances of cells were measured every $5 \mathrm{~min}$ with an input current of $1 \mu \mathrm{A}$ at $16 \mathrm{kHz}$, and the average of normalized resistances was displayed. Arrows indicate the time points of media change. Error bar are indicating the standard deviation of the three different experiment (NGM: normal growth media, $n=5$; NIM: neural induction media, $n=9$; NDM: neural differentiation media, $n=9$ ).

\subsection{Resistance Monitoring of hMSC Layers during Cultivation} with or without Neural Differentiation Factor. We examined the resistance values of the cell layer in 3 different culture media throughout the culture period to determine whether ECIS is useful for real-time monitoring of neural differentiation of hMSCs. Figure 4 shows the resistance values of the cell layer. It was observed that the number of cells attached to the surface of the gold film electrode of the ECIS array naturally increased during the incubation time (data not shown). While time course changes of resistance values were not significantly different between the 3 groups until 80 hrs, the values for NGM and NIM were sharply increased thereafter compared to NDM. The maximal resistance values in NDM were approximately $70 \%$ of those in NGM or NIM at 80 hrs after addition of BHA and DMSO to NGM, which is similar to the time ( $72 \mathrm{hrs}$ ) when the neural marker expression level was significantly increased after BHA and DMSO treatment in a previous study [12]. Overall results indicated that the maximal resistance values of the hMSC layer in the ECIS assay correlated with the degree of neural differentiation.

3.4. Cell Cycle Transition of hMSCs during Neural Differentiation. In principle, impedance values of the cell layer in ECIS can increase as the number of cells and/or adhesion capacity of cells increases. To determine whether relatively lower impedance values observed in the cell layer in NDM compared to those in the other groups were due to lower cell numbers, we examined cell counts in the 3 different media.
As shown in Figure 5(a), the number of cells in NDM was 2.4 times lower than that of the other groups $(P<.05)$. However, cell death rates were not significantly different between the 3 groups (Figure $5(\mathrm{~b})$ ). We performed cell cycle analysis by FACS to determine whether such cell count variations were due to differences in the cell cycle process. As shown in Figure 5(c), G0/G1 cell ratio was higher in cells in NDM than in the other groups $(P<.01)$. These findings indicate that cells in NDM have a delayed cell cycle and are less proliferative, which explains why lower cell counts were obtained in NDM compared to the other media.

\section{Discussion}

Since hMSCs have the capacity to differentiate into cells with a neuronal phenotype in vivo $[13,14]$ or in vitro with specific culture conditions [15-17], they are considered as medically useful materials to treat Parkinson's disease, spinal cord injuries, and optic neural injuries. However, the purity and yield of cells differentiated from stem cells must be sufficient to exert meaningful therapeutic effects. Therefore, monitoring accurate in vitro differentiation prior to transplantation is essential. Noninvasive and real-time monitoring of in vitro differentiation of stem cells may enhance successful stem cell therapy.

In general, stem cell differentiation leads to two types of changes; one is change in expression of differentiationspecific genes and the other is partial or complete inhibition of growth capacity, of cells [18]. Therefore, real-time monitoring of the reduction of growth capacity of stem cells by ECIS would be useful to detect stem cell differentiation.

In the present study, we found that the expression levels of neuron-specific genes, growth capacity, and cell cycle processes of cells cultured in NDM were significantly different from those in the other two media tested when the resistance values of the cell layer in NDM began to differ from the others $(\sim 12 \mathrm{hrs}$ after media change with NDM). In addition, we found that the expression levels of neuron-specific proteins of the cells cultured in NDM were significantly different from those in the other two test media at a time when the resistance value of the cell layer in NDM significantly differed from that of the others $(\sim 72 \mathrm{hrs}$ after media change with NDM, Figure 2). Overall results indicate that cell layer resistance changes reflect the changes in cell number and/or cellular phenotype.

In general, stem cell differentiation is accompanied by a decrease in cell proliferation. Similar to our results, cell number was found to be reduced and cell cycle state was changed during differentiation of MSC into dopaminergic neurons [19].

Our results suggest that the relatively slow changes in resistance values of the neural-differentiating cell layer due to the reduction of growth capacity as well as the morphological changes in long neuron-like cells can be used as a parameter to detect neural differentiation of MSCs. However, a reduction in growth capacity of stem cells does not always indicate differentiation. It may simply indicate cell growth inhibition or increased cell death. To exclude these possibilities and to prove a real neural differentiation of MSCs, examination of 


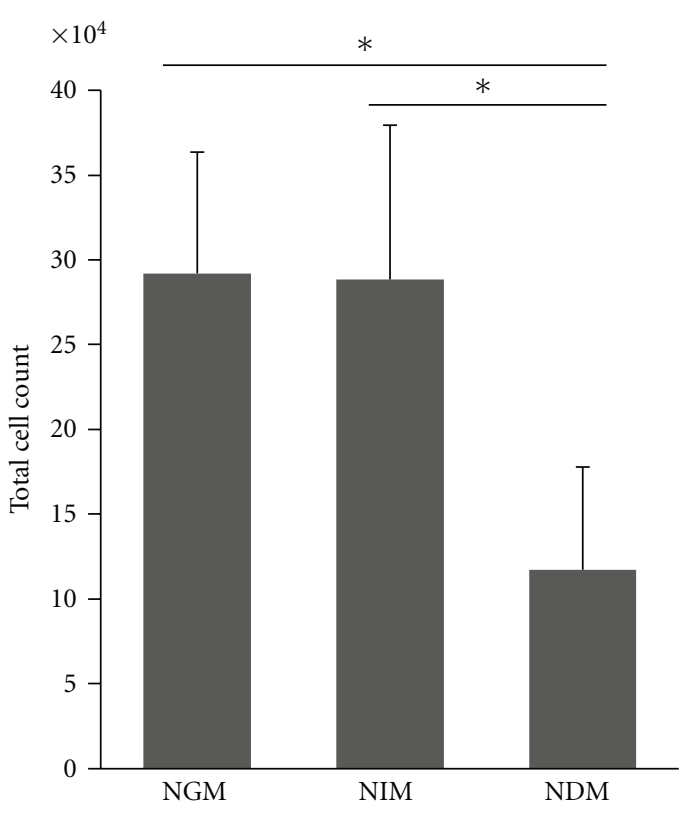

(a)

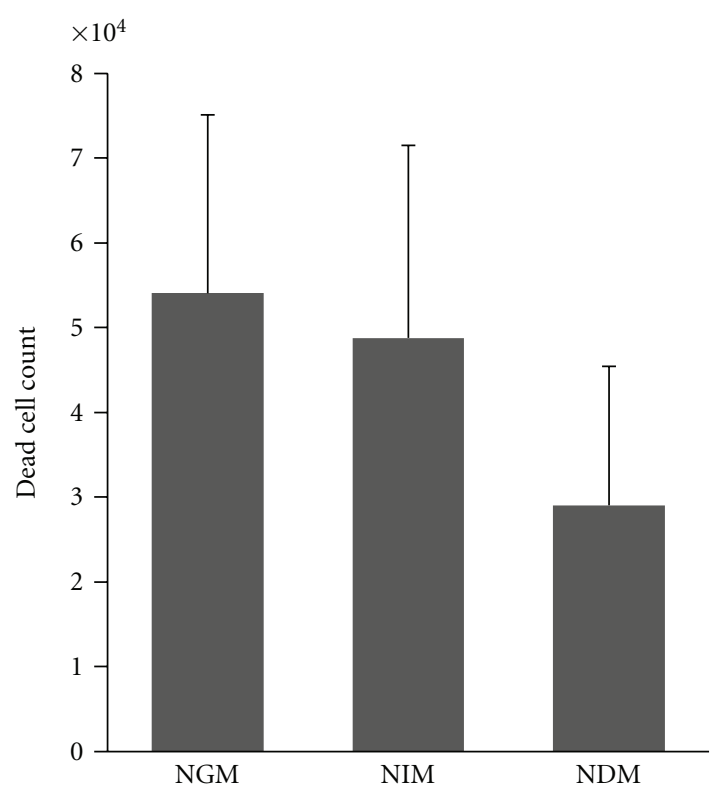

(b)

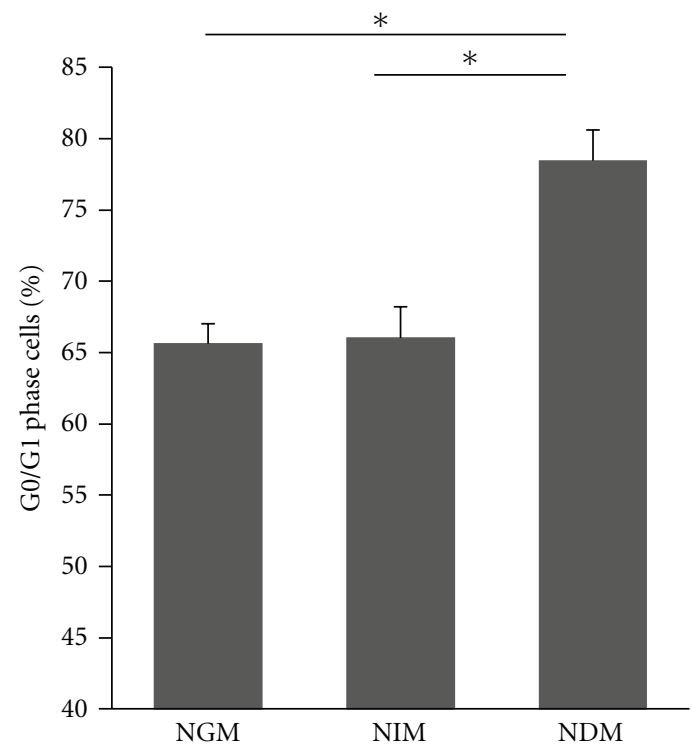

(c)

FIGURE 5: Cell count and cell cycle transition of hMSCs during neural differentiation. (a) Cell counts were determined 36 hr after changing to a NDM (corresponding to $76 \mathrm{hr}$ after culturing in NGM and to $60 \mathrm{hr}$ after changing to a NIM). Data represent means \pm SD $(n=4)$. ${ }^{*} P<.01$. (b) The numbers of death cells were determined by counting the trypan blue stained cells in a hemocytometer. (c) Flow cytometric cell cycle analysis of hMSCs cultured under 3 different culture conditions. DNA content-related cell cycle distribution of hMSCs was determined 24 hr after changing to a NDM. Data represent means \pm SD $(n=6-9) .{ }^{*} P<.01$. NGM: normal growth media; NIM: neural induction media; NDM: neural differentiation media.

their electrophysiological features using in vitro patch clamp recordings is essential. In this regard, current study has some limitation to support that ECIS assay can solely be used for in vitro real-time monitoring of neural differentiation of MSCs.

However, our results suggest that the real-time measurement of cellular electrical resistance by ECIS is useful to determine the optimal time point for performing conventional assays that require end-product for analysis and multiple cell cultures to harvest samples at different time points in a non-real-time manner. This will save samples, reagents, and time used in traditional end-point assays.

\section{Conclusion}

This study suggests that the relatively slow change in resistance values measured by ECIS method can be used as 
a parameter for slowly growing neural-differentiating cells. However, to enhance the competence of ECIS for in vitro real-time monitoring of neural differentiation of MSCs and to exclude the effect of common parameters such as cell number, cell morphology, cell migration, and cytotoxicity, more elaborate experiments will be needed in future studies.

\section{Acknowledgment}

This research was supported by the Pioneer Research Center Program through the National Research Foundation of Korea funded by the Ministry of Education, Science and Technology (no. NRF-2009-0082965).

\section{References}

[1] H. Hentze, R. Graichen, and A. Colman, "Cell therapy and the safety of embryonic stem cell-derived grafts," Trends in Biotechnology, vol. 25, no. 1, pp. 24-32, 2007.

[2] I. Giaever and C. R. Keese, "Micromotion of mammalian cells measured electrically," Proceedings of the National Academy of Sciences of the United States of America, vol. 88, no. 17, pp. 7896-7900, 1991.

[3] I. Giaever and C. R. Keese, "A morphological biosensor for mammalian cells," Nature, vol. 366, no. 6455, pp. 591-592, 1993.

[4] J. H. T. Luong, M. Habibi-Rezaei, J. Meghrous, C. Xiao, K. B. Male, and A. Kamen, "Monitoring motility, spreading, and mortality of adherent insect cells using an impedance sensor," Analytical Chemistry, vol. 73, no. 8, pp. 1844-1848, 2001.

[5] S. Cho and H. Thielecke, "Electrical characterization of human mesenchymal stem cell growth on microelectrode," Microelectronic Engineering, vol. 85, no. 5-6, pp. 1272-1274, 2008.

[6] S. Arndt, J. Seebach, K. Psathaki, H. J. Galla, and J. Wegener, "Bioelectrical impedance assay to monitor changes in cell shape during apoptosis," Biosensors and Bioelectronics, vol. 19, no. 6, pp. 583-594, 2004.

[7] C. Xiao, B. Lachance, G. Sunahara, and J. H. T. Luong, "Assessment of cytotoxicity using electric cell-substrate impedance sensing: concentration and time response function approach," Analytical Chemistry, vol. 74, no. 22, pp. 5748-5753, 2002.

[8] S. Cho, S. Becker, H. von Briesen, and H. Thielecke, "Impedance monitoring of herpes simplex virus-induced cytopathic effect in Vero cells," Sensors and Actuators B, vol. 123, no. 2, pp. 978-982, 2007.

[9] P. Linderholm, T. Braschler, J. Vannod, Y. Barrandon, M. Brouard, and P. Renaud, "Two-dimensional impedance imaging of cell migration and epithelial stratification," Lab on a Chip, vol. 6, no. 9, pp. 1155-1162, 2006.

[10] S. Cho, E. Gorjup, and H. Thielecke, "Chip-based timecontinuous monitoring of toxic effects on stem cell differentiation," Annals of Anatomy, vol. 191, no. 1, pp. 145-152, 2009.

[11] C. Hildebrandt, H. Büth, S. Cho, . Impidjati, and H. Thielecke, "Detection of the osteogenic differentiation of mesenchymal stem cells in 2D and $3 \mathrm{D}$ cultures by electrochemical impedance spectroscopy," Journal of Biotechnology, vol. 148, no. 1, pp. 83-90, 2010.

[12] K. E. Mitchell, M. L. Weiss, B. M. Mitchell et al., "Matrix cells from Wharton's jelly form neurons and glia," Stem Cells, vol. 21, no. 1, pp. 50-60, 2003.
[13] T. R. Brazelton, F. M. V. Rossi, G. I. Keshet, and H. M. Blau, "From marrow to brain: expression of neuronal phenotypes in adult mice," Science, vol. 290, no. 5497, pp. 1775-1779, 2000.

[14] K. Nakano, M. Migita, H. Mochizuki, and T. Shimada, "Differentiation of transplanted bone marrow cells in the adult mouse brain," Transplantation, vol. 71, no. 12, pp. 1735-1740, 2001.

[15] J. Sanchez-Ramos, S. Song, F. Cardozo-Pelaez et al., "Adult bone marrow stromal cells differentiate into neural cells in vitro," Experimental Neurology, vol. 164, no. 2, pp. 247-256, 2000.

[16] J. Kohyama, H. Abe, T. Shimazaki et al., "Brain from bone: efficient "meta-differentiation" of marrow stroma-derived mature osteoblasts to neurons with Noggin or a demethylating agent," Differentiation, vol. 68, no. 4-5, pp. 235-244, 2001.

[17] A. R. Alexanian, D. J. Maiman, S. N. Kurpad, and T. A. Gennarelli, "In vitro and in vivo characterization of neurally modified mesenchymal stem cells induced by epigenetic modifiers and neural stem cell environment," Stem Cells and Development, vol. 17, no. 6, pp. 1123-1130, 2008.

[18] M. Andreeff, D. W. Goodrich, and A. B. Pardee, "Cell proliferation, differentiation, and apoptosis," in Holland-Frei Cancer Medicine, Section 1 Cancer Biology, chapter 2, pp. 1732, BC Decker, 5th edition, 2000.

[19] K. A. Trzaska, E. V. Kuzhikandathil, and P. Rameshwar, "Specification of a dopaminergic phenotype from adult human mesenchymal stem cells," Stem Cells, vol. 25, no. 11, pp. 27972808, 2007. 

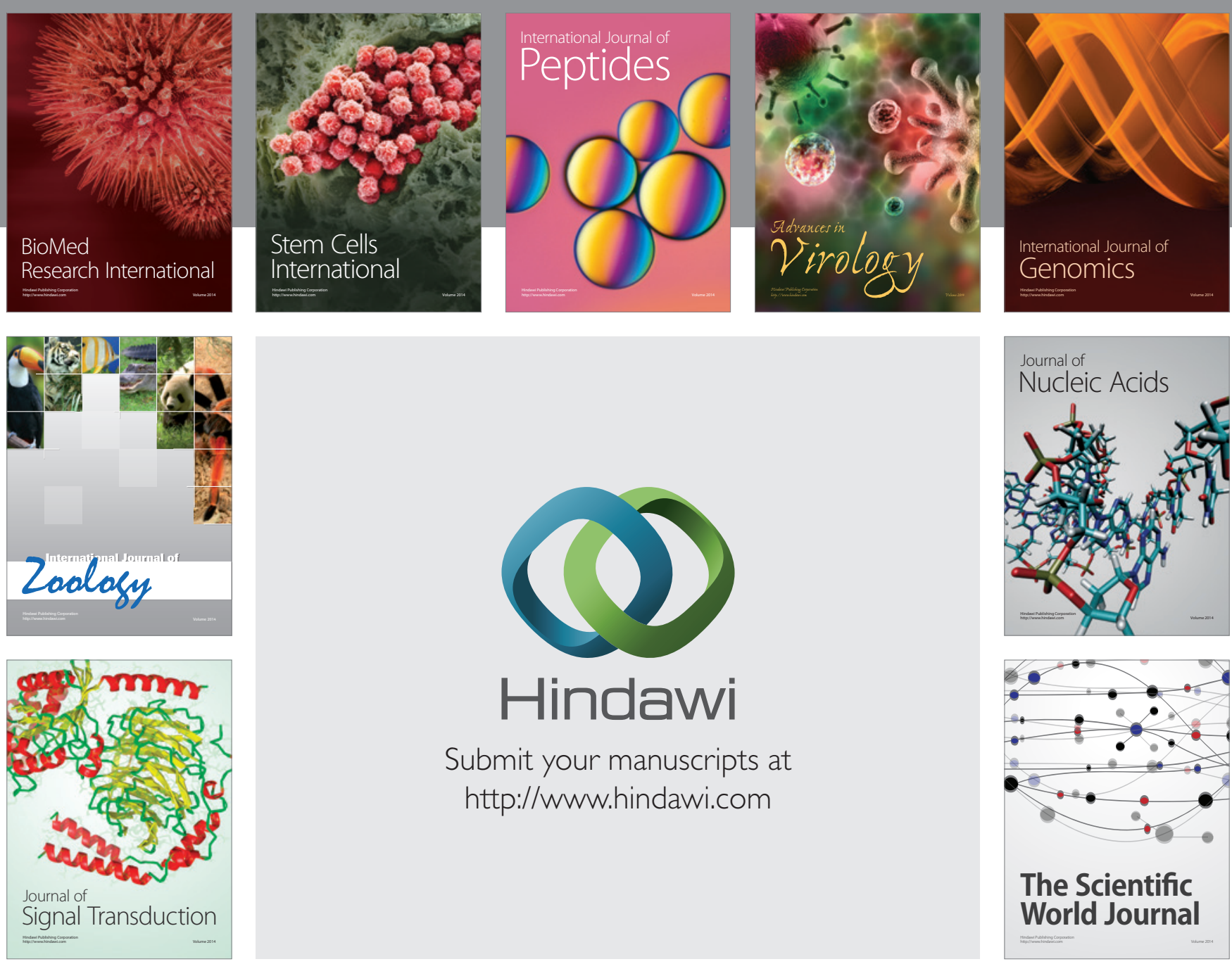

Submit your manuscripts at

http://www.hindawi.com
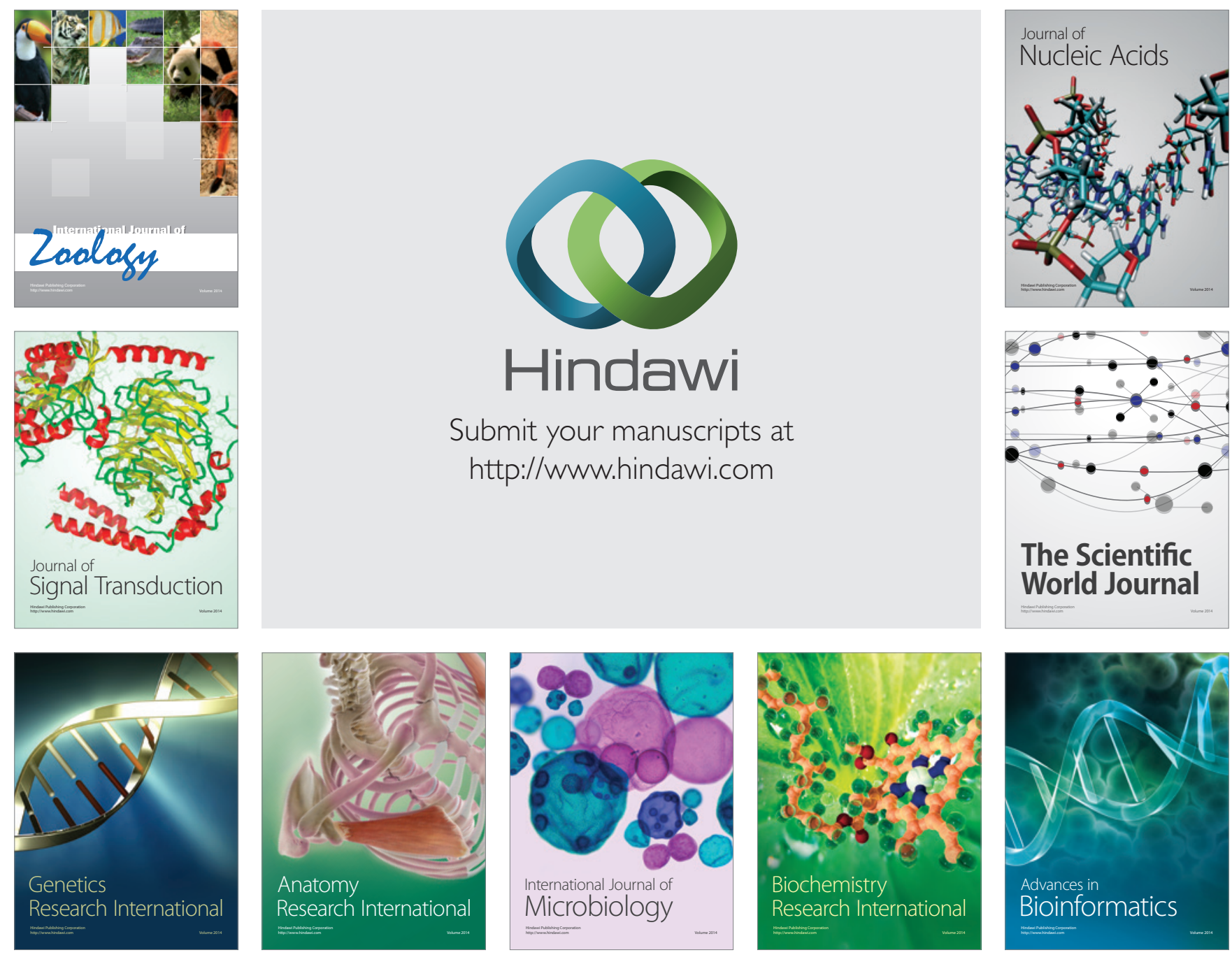

The Scientific World Journal
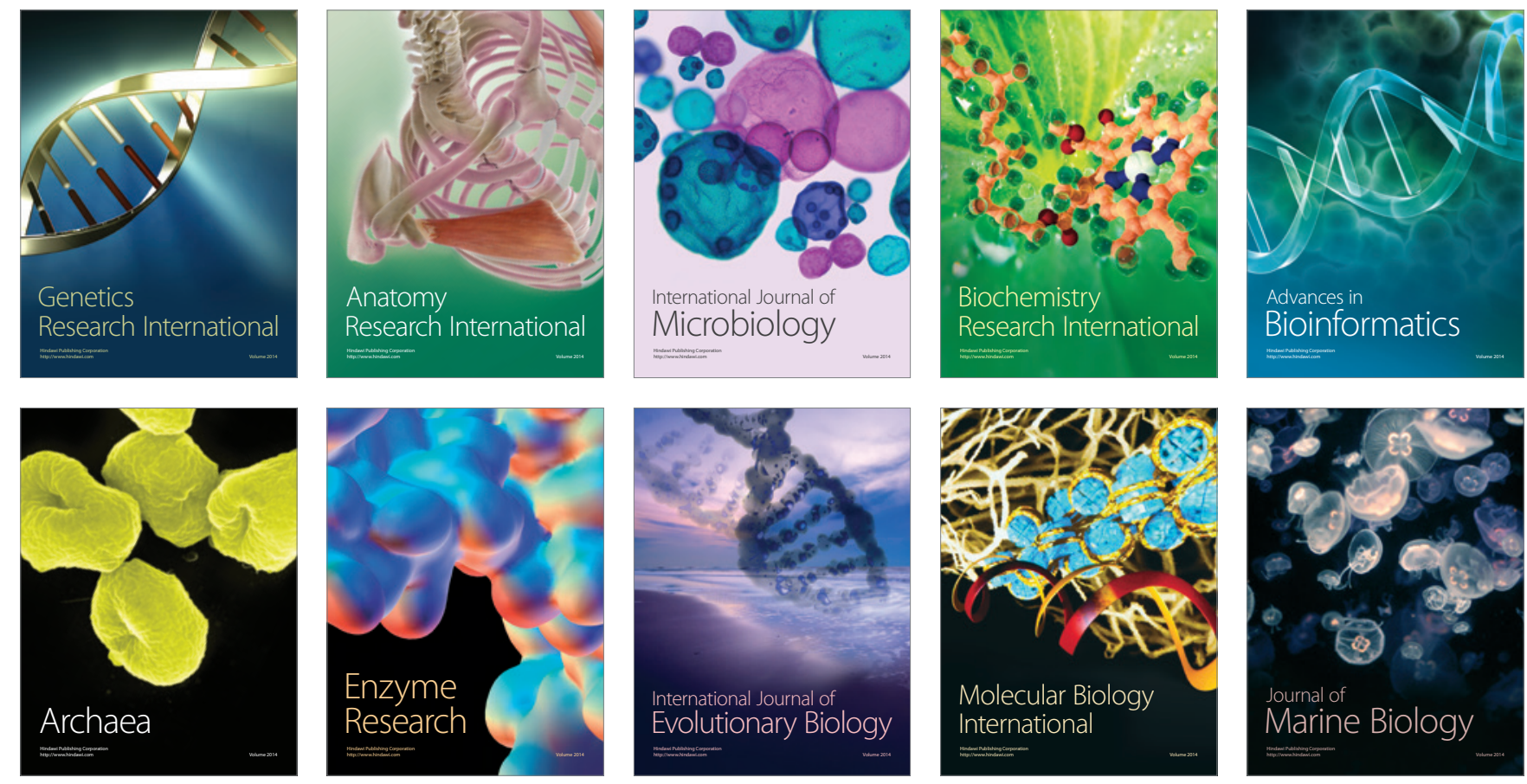